\title{
Patient-specific computational haemodynamics associated with surgical creation of an arteriovenous fistula
}

George Hyde-Linaker

University of Strathclyde

Pauline Hall Barrientos

Queen Elizabeth University Hospital

Sokratis Stoumpos

Queen Elizabeth University Hospital

Asimina Kazakidi ( $\square$ asimina.kazakidi@strath.ac.uk)

University of Strathclyde https://orcid.org/0000-0001-7124-4123

\section{Research Article}

Keywords: computational fluid dynamics, haemodynamics, chronic kidney disease, arteriovenous fistula, STAR-CCM+

Posted Date: June 18th, 2021

DOl: https://doi.org/10.21203/rs.3.rs-627689/v1

License: (9) This work is licensed under a Creative Commons Attribution 4.0 International License. Read Full License 


\title{
Patient-specific computational haemodynamics associated with surgical creation of an arteriovenous fistula
}

\author{
George Hyde-Linaker ${ }^{1}$, Pauline Hall Barientos ${ }^{2}$, Sokratis Stoumpos ${ }^{3,4}$, and Asimina \\ Kazakidi $^{1 *}$ \\ ${ }^{1}$ Department of Biomedical Engineering, University of Strathclyde, Glasgow, UK \\ ${ }^{2}$ Image Centre of Excellence, Queen Elizabeth University Hospital, Glasgow, UK \\ ${ }^{3}$ Renal and Transplant Unit, Queen Elizabeth University Hospital, Glasgow, UK \\ ${ }^{4}$ Institute of Cardiovascular and Medical Sciences, BHF Glasgow Cardiovascular \\ Research Centre, University of Glasgow, Glasgow G12 8TA, UK \\ *Correspondence: asimina.kazakidi@strath.ac.uk
}

\section{Abstract}

Despite arteriovenous fistulae (AVF) being the preferred vascular access for haemodialysis, high primary failure rates (30-70\%) and low one-year patency rates $(40-70 \%)$ hamper their use. The haemodynamics within the vessels of the fistula change significantly following surgical creation of the anastomosis and can be a surrogate of AVF success or failure. Computational fluid dynamics (CFD) can crucially predict AVF outcomes through robust analysis of a fistula's haemodynamic patterns, which is impractical in-vivo. We present a proof-of-concept CFD framework for characterising the AVF blood flow prior and following surgical creation of a successful left radiocephalic AVF in a 20-year-old end-stage kidney disease patient. The reconstructed vasculature was generated utilising multiple contrast-enhanced magnetic resonance imaging (MRI) datasets. Large eddy simulations were conducted for establishing the extent of arterial and venous remodelling. Following anastomosis creation, a significant 2-3-fold increase in blood flow rate was induced downstream of the left subclavian artery. This was validated through comparison with post-AVF patient-specific phase-contrast data. The increased flow rate yielded an increase in time-averaged wall shear stress (TAWSS), a key marker of adaptive vascular remodelling. We have demonstrated TAWSS and oscillatory shear distributions of the transitional-flow in the venous anastomosis are predictive of AVF remodelling.

Keywords: computational fluid dynamics, haemodynamics, chronic kidney disease, arteriovenous fistula, STAR-CCM+ 


\section{Introduction}

In 2017, the global prevalence of chronic kidney disease (CKD) was $9.1 \%$, which is roughly 700 million cases. ${ }^{10}$ Patients with CKD experience a gradual decline in renal function to the extent that renal replacement therapy (RRT) is required for survival in end-stage kidney disease (ESKD). Over $85 \%$ of the global ESKD population is haemodialysis (HD) dependent ${ }^{27}$ requiring a reliable access to the systemic circulation to achieve mechanical blood filtering and replace the kidney function. The preferred vascular access for ESKD patients is an arteriovenous fistula (AVF), a native vessel segment created by surgically joining a vein and an artery, commonly in the patient's non-dominant arm. ${ }^{6}$ Creation of an AVF establishes a shunt from the high pressure, low capacitance arterial system to the low pressure, high capacitance venous system ${ }^{16}$ causing an increase in the arterial diameter and the 'arterialisation' (thickening and increased elasticity) of the vein. This process takes approximately 6-8 weeks ${ }^{31}$ and, when successful, permits consistently high blood flow rates in excess of $600 \mathrm{~mL} / \mathrm{min}^{2}$ whilst providing a durable vascular access for delivery of efficient and repeatable dialysis. A major problem with AVF is the high frequency of primary failure as a result of either poor maturation or early thrombosis. As such, vascular access dysfunction is one of the leading causes of morbidity and mortality among ESKD patients. ${ }^{13,30}$ Neointimal hyperplasia $(\mathrm{NH})$ and inadequate outward venous remodelling are the two leading causes of AVF failure. ${ }^{36}$ The exact mechanisms behind these processes remain mostly unknown, highlighting the need for exploring the haemodynamic environment caused by vascular access surgery.

Computational fluid dynamics (CFD) is a useful tool that can be used to assess patientspecific haemodynamic predictors of AVF success or failure ${ }^{32}$ beyond the traditional demographic, clinical and biological predictors. The haemodynamics and wall shear stress (WSS) influencing the AVF environment are difficult to measure in-vivo. CFD allows a wide range of calculations into such haemodynamic metrics. ${ }^{39}$ This is key for assessing regions of patient-specific anatomical characteristics that are susceptible to abnormal WSS distributions and disturbed flow. In this study, we present a detailed $3 \mathrm{D}$ reconstruction of the arterial vasculature from the aorta to the radial artery, which is the preferred site for AVF creation. ${ }^{22}$ This is based on ferumoxytol-enhanced magnetic resonance angiography (FeMRA) data used in conjunction with a CFD 
framework. Ferumoxytol is a superparamagnetic iron oxide nanoparticle preparation, developed firstly as a contrast agent in $2000 .{ }^{33}$ The use of FeMRA avoids the risks that historic contrast-enhanced methods pose in ESKD patients. For example, gadolinium-based contrast agents (GBCAs) are linked with a risk of nephrogenic systemic fibrosis in ESKD.

The haemodynamics of the 3D construction generated from multiple FeMRA scans were simulated using an established finite-volume implementation in STAR-CCM+. Large eddy simulations (LES) were completed using a wall-adapting local-eddy (WALE) viscosity subgrid scale model. The patient was 20 years old with a side-toend radiocephalic AVF and was scanned before surgery and six weeks after AVF creation. The aims of this study were two-fold: (i) to simulate the patient haemodynamics from the aorta to the radial artery (proximal to the anastomosis) priorto and post-AVF creation and (ii) to simulate the site-specific haemodynamics at the radiocephalic anastomosis under the non-physiological conditions induced by the AVF. The boundary conditions in this case were defined as per phase-contrast FeMRA data. This simulation informed the WSS distribution at the peri-anastomotic region and the velocity profile in the proximal artery and venous outflow segments.

\section{Materials and methods}

The presented framework produces a singular meshed domain of the patient-specific vasculature from the ascending aorta to the arteries of the left forearm using three FeMRA-obtained DICOM stacks per visit. The haemodynamics of the patient-specific vasculature produced from the framework were subsequently simulated using CFD after discretising (meshing) the domain (Fig.1). 


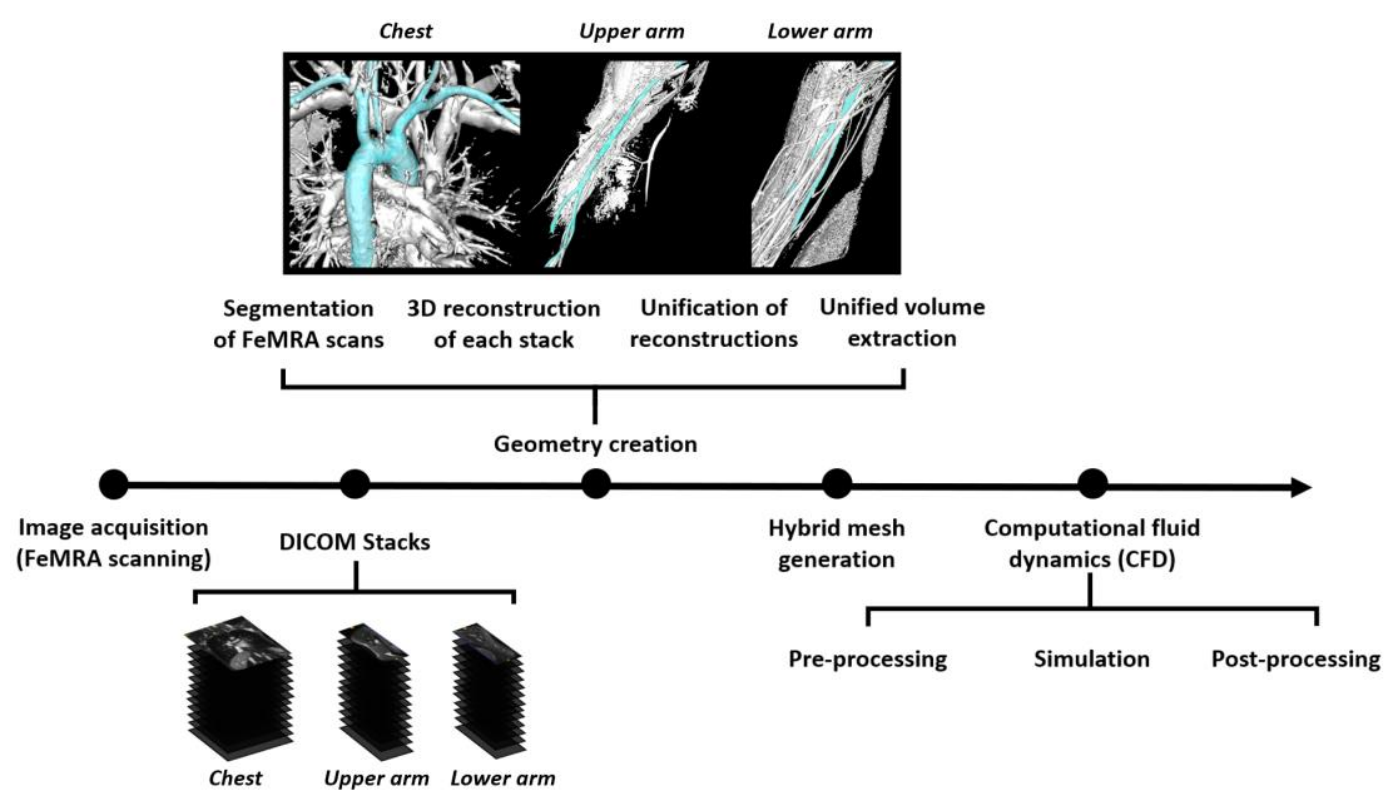

Figure 1. Proposed framework for the segmentation, reconstruction, geometry unification and volume extraction of the patient-specific geometry based on ferumoxytol-enhanced magnetic resonance angiography (FeMRA) multi-stack images and subsequent CFD analysis.

\section{FeMRA DICOM stacks}

Due to the differing scales of the vasculature in this study, three separate scans for the chest, left upper arm, and left lower arm were acquired (Fig. 1). This is due to the varying resolutions required when scanning smaller vessels as opposed to larger ones (Supplementary Material). All scans were performed on a 3.0T Prisma MRI scanner (Magnetom, Siemens Medical Solutions) with local phased-array imaging coils using a standardised protocol similar to that of standard MRA studies with GBCAs. All patients were imaged in the supine position. ${ }^{33}$

\section{Segmentation, reconstruction and computational domain}

The three FeMRA datasets (Fig. 1) were segmented, reconstructed and united into a singular meshed geometry using boolean techniques in Autodesk Meshmixer@, a specialised opensource software for manipulating 3D meshes, as follows:

A. Vessel identification: The regions of interest for each of the DICOM stacks were initially visualised using OsiriX (Pixmeo, Fig. 1). The critical areas to assess visually were the common bifurcations of i) the body and upper arm FeMRA scans, and ii) the upper arm and lower arm scans (Fig. 2a). The two respective regions selected were the deep brachial artery bifurcation and the radial-ulnar bifurcation. 
B. Segmentation and reconstruction: Each DICOM stack was segmented and reconstructed 'semi-automatically' using open-source ITK-SNAP algorithms (www.itksnap.org). This involved manual editing following automatic segmentations that used a common threshold ratio. ${ }^{38}$ Unstructured surface meshes of the reconstructions were exported in a stereolithographic (STL) file format.

C. Assembly of reconstructions: The three separate reconstructions from the chest, upper and lower arm scans were imported into Meshmixer to be united into a single STL surface mesh. They were manipulated so that the common areas (the deep brachial artery bifurcation and the radial-ulnar bifurcation) overlapped (Fig. 2a), to ensure any duplicated regions were clipped. The geometries were then combined using a 'Boolean Union' function. The areas of overlap were smoothed to preserve the dimensions of the geometry whilst ensuring the transition from one reconstruction to the other was valid anatomically. The dimensions of the STL surface mesh were compared to the initial DICOM files at various intervals for verification.

D. Hole filling and surface remeshing: The 'Hole Filler' tool in OpenFlipper (RWTH Aachen) was used for resolving any areas of missing elements. The new geometry was remeshed with isotropic elements to improve the mesh quality of the transition between the original reconstructions, and then smoothed with a low-level smoothing factor of 2.

E. Domain clipping: The inlet and outlets of the domain were subsequently clipped (normal to the vessel centreline) in Paraview (http://www.paraview.org), at distances of $0.5 \mathrm{D}$ and $1.5 \mathrm{D}$ respectively, where $\mathrm{D}$ is the diameter of each respective patch. Centrelines were computed utilising the VMTK libraries (http://www.vmtk.org).

F. Second surface remeshing, smoothing and geometry check: The clipped surface mesh was smoothed and remeshed iteratively in OpenFlipper to ensure the mesh quality was sufficient at each bifurcation. This prevents the dimensions of the geometry from being distorted in future polyhedral volume meshing. The geometry was then compared with the original DICOM images to ensure no part of the process distorted or inappropriately scaled the meshes.

G. Computational domain generation: The final step in the framework involved the generation of the computational domain, which here was completed in STAR-CCM+ (Siemens PLM). The clipped inlet and outlet boundaries of the geometry were closed normal to the centreline to define its volume (Fig. 2b), before proceeding with the hybrid mesh generation described in the next section. 


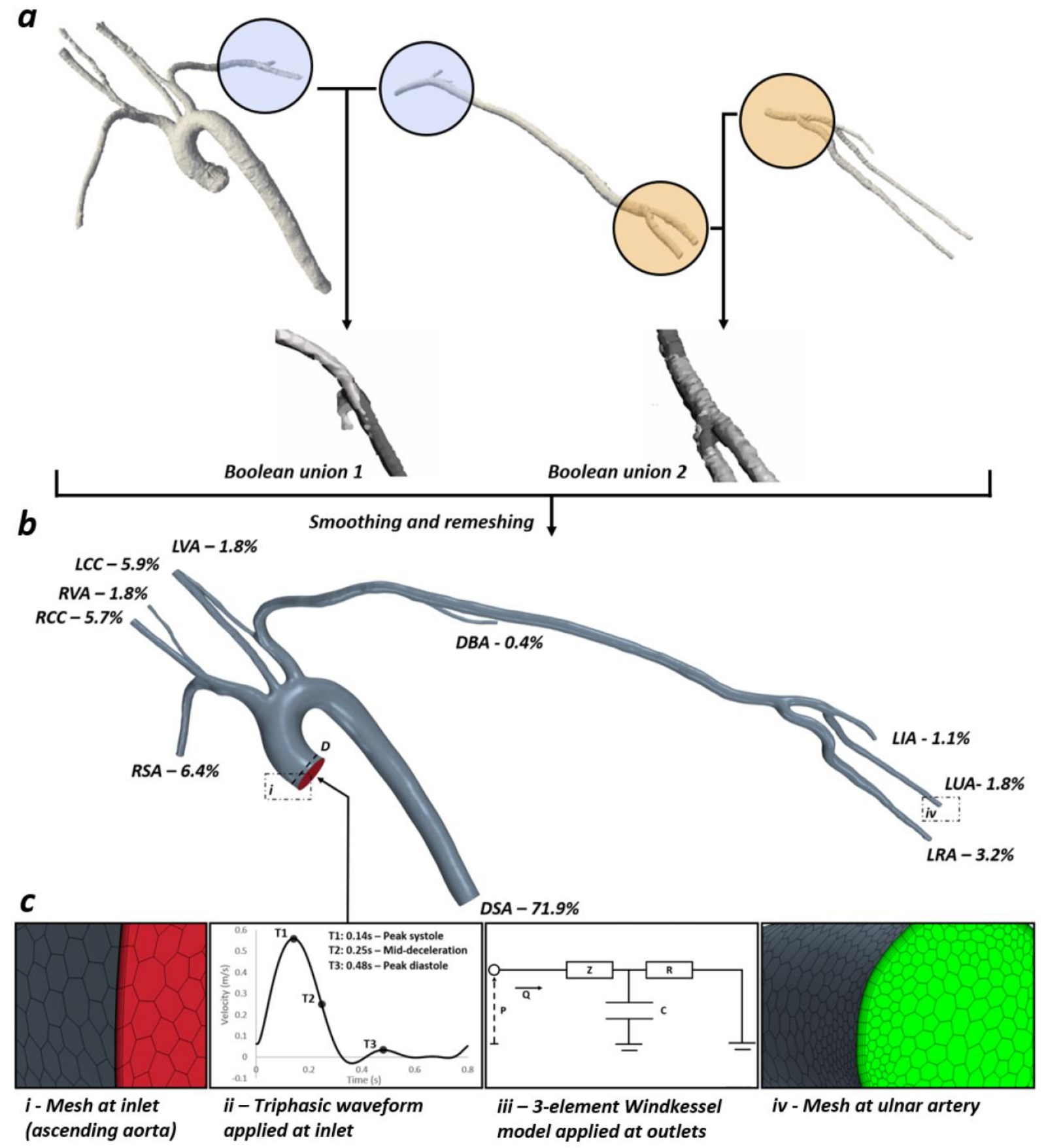

Figure 2: (a) Unification of FeMRA-based reconstructions. (b) Smoothed post-surgery geometry with the pre-AVF cardiac output percentage of each branch given in italics. (c) (i) Meshed geometry at the aortic inlet, (ii) velocity inlet waveform, (iii) lumpedparameter three-element Windkessel model, and (iv) mesh at the ulnar artery outlet. RSA: Right subclavian artery; RCC: Right common carotid; RVA: Right vertebral artery; LCC: Left common carotid; LVA: Left vertebral artery; DBA: Deep brachial artery; LIA: Left interosseous artery; LUA: Left ulnar artery; LRA: Left radial artery DA: Descending aorta.

\section{Hybrid mesh generation}

A hybrid mesh with prism layers and polyhedral elements was used for discretising the computational domain. Polyhedral meshes contain approximately five times fewer 
cells than a comparable tetrahedral mesh and are less sensitive to stretching. Prism layers were incorporated to reduce numerical diffusion adjacent to the wall and capture viscous effects. The thickness of the first prism layer, $y$, was calculated assuming a $y+$ value of $1,{ }^{18}$ given by:

$$
y+=\frac{\rho . u_{T} \cdot y}{\mu}
$$

where $\rho$ is the fluid density, $u$ T is the friction velocity, and $\mu$ is the dynamic viscosity of the fluid.

Following the calculation of the initial prism layer height, a mesh independence study was completed. The number of prism layers and cell base size was varied, whilst the prism layer total thickness was kept constant. The most suitable mesh had 8 prism layers and base size of $3.8 \mathrm{E}-4 \mathrm{~m}$, resulting in a hybrid mesh with 3776730 elements and a maximum $\mathrm{y}+$ value of 0.36 . The mean grid convergence index $(\mathrm{GCl})^{7}$ at 16 locations in the vasculature was computed as 0.99 , which was deemed appropriate. The mesh characteristics were also validated for LES by computation of the maximum wall y+, Taylor Mirco-, and Kolmogorov length scales (Supplementary Material).

\section{Numerical modelling \\ Models}

LES computes the largest scales of turbulent motion, with the smallest scales being modelled with a WALE subgrid scale approach in this study. The LES governing equations were obtained by filtering the time-dependent 3D incompressible NavierStokes momentum and continuity equations, which become:

$$
\begin{gathered}
\frac{\partial \bar{u}_{i}}{\partial x_{i}}=0 \\
\frac{\partial \bar{u}_{i}}{\partial t}+\frac{\partial}{\partial x_{j}}\left(\bar{u}_{i} \bar{u}_{j}\right)=-\frac{1}{\rho} \frac{\partial p}{\partial x_{i}}+v \frac{\partial}{\partial x_{j}}\left(\frac{\partial \bar{u}_{i}}{\partial x_{j}}+\frac{\partial \bar{u}_{j}}{\partial x_{i}}\right)-\frac{\partial \tau_{i j}}{\partial x_{j}}
\end{gathered}
$$

where $\bar{u}$ is the velocity vector, $\mathrm{p}$ is the pressure, and $\mathrm{T}_{\mathrm{ij}}$ denotes subgrid-scale stresses.

LES investigations were based on an implicit unsteady finite volume model in Star$\mathrm{CCM}+$ with a timestep of $0.001 \mathrm{~s}$. Optimised 2 nd-order temporal discretisation was employed, which reduces the leading-order truncation error by a factor of 2, compared to base-level 2nd-order temporal discretisation. 


\section{Continua definition and haemodynamic metrics}

In the 'proximal-to-fistula' simulation, blood was assumed to be a Newtonian fluid $\left(1060 \mathrm{kgm}^{-3}, 0.0035 \mathrm{~Pa} . \mathrm{s}\right)$. This common assumption omits the 'shear-thinning' property of blood, which manifests at shear rates below $50-100 \mathrm{~s}^{-1}$. This assumption is reasonable due to the significant exposure to high shear throughout the domain during the cardiac cycle, which suppresses the rouleaux formation. A generalised CarreauYasuda non-Newtonian model ${ }^{4}$ was used in the juxta-anastomosis simulation, due to the small arterial diameters producing shear rates that would stretch the Newtonian rheology assumption. The continua definition influences the haemodynamic parameters of interest, the time-averaged WSS (TAWSS) and oscillatory shear index (OSI). 19,20,35 TAWSS evaluates shear stress exerted on the vessel walls during the cardiac cycle (Equation 4), whereas OSI, assesses the directional change of WSS during the cardiac cycle (Equation 5), where $t$ is the time, $T$ is the cardiac cycle time, and $\tau_{w}$ is the wall shear stress.

$$
\begin{gathered}
\text { TAWSS }=\frac{1}{T} \int_{0}^{T}\left|\tau_{w}\right| d t \\
O S I=\frac{1}{2}\left(1-\frac{\left|\int_{0}^{T} \tau_{w} d t\right|}{\int_{0}^{T}\left|\tau_{w}\right| d t}\right)
\end{gathered}
$$

\section{Boundary conditions}

In the proximal-to-fistula simulations, a triphasic velocity inlet waveform (Figure 3C-ii) was assigned at the aortic root, calculated as a polynomial using previous data. ${ }^{1}$ Patient-specific phase-contrast flow rate data was used for prescribing the velocity inlet waveform in the radial artery for the juxta-anastomosis simulation. A no-slip rigid wall condition was assumed in all simulations. In the proximal-to-fistula simulations the 10 pressure outlets were coupled to lumped-parameter three-element Windkessel (WK) models (Figure 2c-iii). The capacitance (C) and resistance (R) parameters account for the compliance and peripheral resistance of the terminal branch, respectively, while the characteristic impedance element $(Z)$ represents the resistance of the terminal vessel.

The expression relating flow rate and pressure in the 3-element WK model is as follows: 


$$
\frac{\partial p}{\partial t}+\frac{p}{R C}=\frac{Q}{C}\left(1+\frac{Z}{R}\right)+Z\left(\frac{\partial Q}{\partial t}\right)
$$

The discretisation of this model in the time-domain for purposes of a time-discretised CFD study, yields:

$$
p^{n+1}=\frac{\frac{\tau}{\Delta t} p^{n}+Q^{n+1}\left(R+Z\left(1+\frac{\tau}{\Delta t}\right)\right)-Z \frac{\tau}{\Delta t} Q^{n}}{1+\frac{\tau}{\Delta t}}
$$

where $\mathrm{T}=\mathrm{RC}, \mathrm{p}$ and $\mathrm{p}^{\mathrm{n}+1}$ are the pressure at the current and next timestep, $\mathrm{Q}$ and $\mathrm{Q}^{\mathrm{n}+1}$ are the volumetric flow rates at the current and next timestep, and $\Delta t$ is the timestep.

The use of 3-element WK models produced physiologically relevant pressure values $(120 / 80 \mathrm{mmHg})$ in the proximal-to-fistula simulation at the descending aorta outlet. This was compared with the 'splitting method'9 $(22 /-8 \mathrm{mmHg})$, which involved computing branch diameters immediately following a bifurcation (see Supplementary Material). The capacitance and resistive values of each 3-element WK model are dependent on the artery's characteristics in addition to the distal vasculature. In the post-AVF proximal-to-fistula case, the radial artery outlet was prescribed as a pressure waveform, calculated using predefined $R C$ values interpolated from literature $(R=$ 1.05E5 Pa.s. $\left.\mathrm{m}^{-3}, \mathrm{C}=3.25 \mathrm{E}-6 \mathrm{~m}^{3} \mathrm{~Pa}^{-1}\right)^{15}$, and patient-specific phase-contrast data. Notwithstanding this exception, an estimate for the characteristic impedance $Z$ and capacitance $\mathrm{C}_{0}$ of each terminal artery is given by:

$$
\begin{gathered}
Z=\frac{\rho_{f} c_{d}}{A_{d}} \\
C_{0}=\frac{A_{d} L}{\rho\left(c_{d}\right)^{2}}
\end{gathered}
$$

where $L, C_{d}$, and $A_{d}$ are the vessel length, pulse wave velocity (PWV) and vessel area at diastolic pressure, respectively. Characteristic $R$ and $C$ values for each 3-element WK model can subsequently be calculated with the methodology detailed in Alastruey ${ }^{3}$, using Equations 10-11: 


$$
\begin{gathered}
R=\left(\frac{1}{1-\frac{\lambda}{2 \varphi^{4}}}-1\right) Z=\left(\frac{\lambda}{2 \varphi^{4}-\lambda}\right) Z \\
C=\sum C_{T_{n}}=\left(\frac{1}{1-2 \lambda \varphi^{3}}-1\right) C_{0}=\left(\frac{2 \lambda \varphi^{3}}{1-2 \lambda \varphi^{3}}\right) C_{0}
\end{gathered}
$$

where $\lambda=0.7$ and $\phi=\sqrt{ } 0.6$ are constants based on prior data ${ }^{29}$.

\section{Results}

The results are presented in three parts: a geometrical characterisation of the pre- and post-fistula geometries is described first (Fig. 3), an analysis of the global haemodynamics from the aorta to the radial artery (proximal-to-fistula simulation) is provided second (Figs. 4-6), followed by the localised flow development in the remodelled patient-specific arteriovenous fistula, 6-weeks post-surgery (Fig. 7-8) at three intervals of the cardiac cycle: peak systole (T1), mid-deceleration (T2), and peak diastole (T3).

\section{Pre- and post-fistula geometry characterisation}

In order to characterise the geometries, the centrelines of each computational domain were computed utilising the VMTK libraries (http://www.vmtk.org). Utilising the methodology described previously, the pre- and post-surgery surface area of the vasculature was compared (Fig. 3a). Based on the vessels' centreline, from the ascending aorta (inlet) to the descending aorta and the left radial artery, the curvature (Fig. 3b) and tortuosity were also estimated. Minor differences were observed between the geometries (Table 1).

Table 1: Pre- and post-AVF geometry characterisation

\begin{tabular}{|l|l|l|c|}
\hline Metric & Pre-operative & Post-surgical & $\%$ difference \\
\hline Total surface area $\left(\mathrm{m}^{2}\right)$ & $3.9478 \mathrm{E}-02$ & $3.6875 \mathrm{E}-02$ & $6.5 \%$ \\
\hline AscA-LRA Mean Tortuosity & 18.06 & 20.06 & $11 \%$ \\
\hline AscA-LRA Tortuosity Standard Deviation & 15.46 & 17.23 & $11 \%$ \\
\hline
\end{tabular}




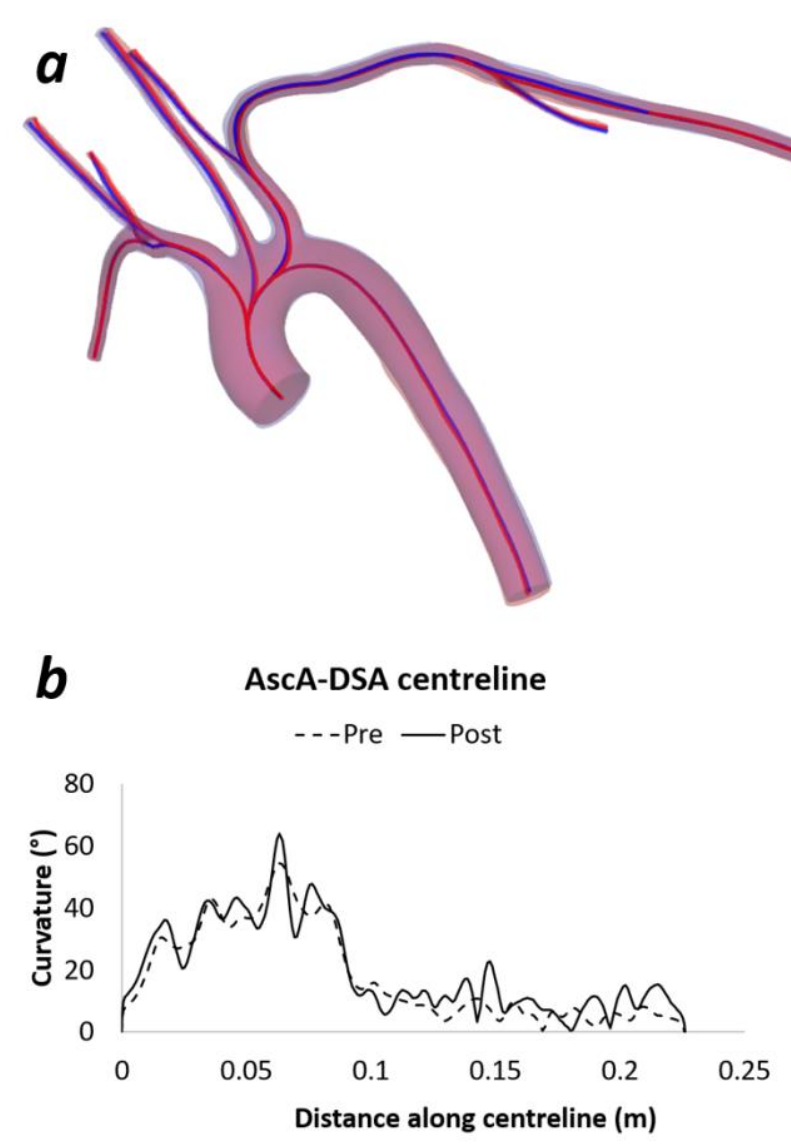

Blue region - Pre-AVF reconstruction

Red region - Post-AVF reconstruction

- Pre-AVF centreline

- Post-AVF centreline

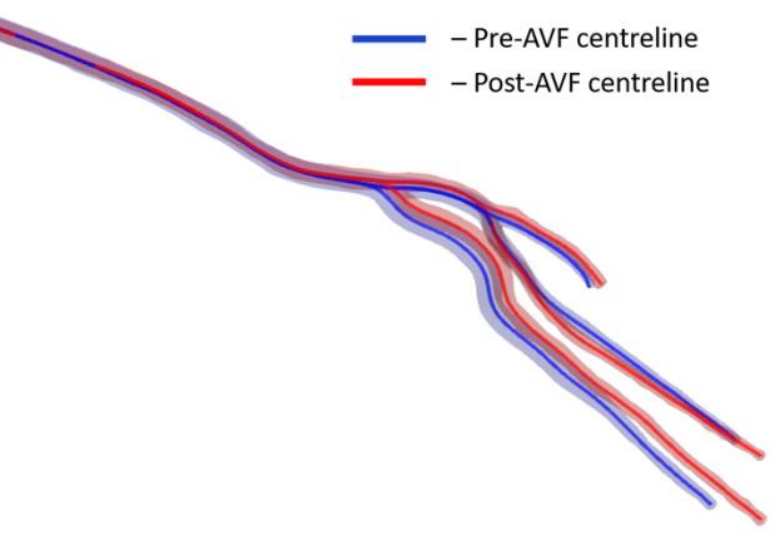

AscA-LRA centreline

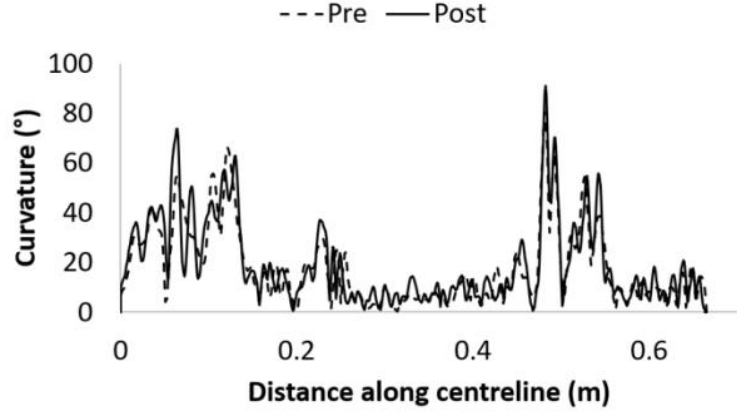

Figure 3: (a) Pre- and post-AVF reconstructions, and centreline curvature plots from (b) ascending aorta (AscA) to descending aorta (DSA) and left radial artery (LRA) outlets.

\section{Aorta-to-radial artery haemodynamics}

Fig. 4a-b demonstrates the changes in the haemodynamic environment proximal to the fistula for the pre- and post-AVF vasculatures through velocity streamlines at three instances during the cardiac cycle (T1-T3). The post-AVF case reflects the decrease in peripheral resistance and increased flow rate in the radial artery. It is noted that the geometry in Fig. 4b does not include the fistula, due to the aforementioned limits of the Newtonian rheology assumption and the necessity of differing mesh criterion between the cases. The evolution of the haemodynamics in both circumstances (Fig. 4a-b) reveals a decrease in the velocity magnitude in the thoracic aorta at the observed intervals following peak systole. Pre-operatively, flow velocity magnitudes in excess of $1.6 \mathrm{~m} / \mathrm{s}$ (local $\operatorname{Re}_{\max } \approx 3906$ ) were observed at T1-T3 in the left arm in the subclavian artery adjacent to the deep brachial artery bifurcation. In the post-surgery case, markedly increased velocity magnitudes (local $R_{\max } \approx 4762$ ) were found throughout the left arm, notably the left-subclavian, brachial, and radial arteries (Fig. 4b). In-plane vector patterns at peak systole (Fig. 4c) exhibited many similarities between the pre- 
and post-AVF cases up to the radial-ulnar bifurcation (slices $\alpha-\delta, \alpha^{\prime}-\delta^{\prime}$ ) and the velocity contours indicated comparable flow skewing directions, albeit with higher velocities post-surgery. However, downstream of the radial-ulnar bifurcation (slices $\varepsilon-\zeta, \varepsilon^{\prime}-\zeta$ ), the generation of secondary vortices differed in pattern and location pre- and post-AVF, while blood flow appeared more skewed post-operatively (cranially in slice $\zeta^{\prime}$ ).

Fig. 5a-b presents the comparative time-averaged haemodynamic metrics (TAWSS and OSI) for the pre- and post-AVF vasculatures. Pre-AVF, high TAWSS values (in excess of 20Pa) were observed in localised patches at three notable locations: the left subclavian and deep brachial arteries, and at a small region of the radial-ulnar bifurcation. Post-AVF, the distribution of high TAWSS values $(>20 \mathrm{~Pa})$ in the left subclavian artery was much more pronounced and extended; conversely, the deep brachial artery exhibited significantly lower TAWSS values, while there was a distinct increase in TAWSS (> 20Pa) along the brachial and radial arteries and decrease in the ulnar artery. Minor differences were observed in the respective OSI contour plots (Fig. 5-right), with the highest OSI magnitudes being found in the aortic arch and thoracic aorta. Additionally, moderate values of OSI $(\approx 0.2)$ were observed in close proximity of the pre-surgery radial-ulnar bifurcation, which changed in distribution following AVF creation. To validate the imposed boundary condition for the radial artery outlet in the post-AVF case, the flow rate in the radial artery (slice $\varepsilon^{\prime}$ in Fig. 4c) was compared to phase-contrast FeMRA data of the 20-year-old patient (Fig. 6). The flow rates predicted by the CFD model demonstrate a maximum percentage difference of $6.93 \%$ at peak systole ( $\mathrm{T} 1$ ) and a mean difference of $1.48 \%$ between the volumetric flowrate integrals. 

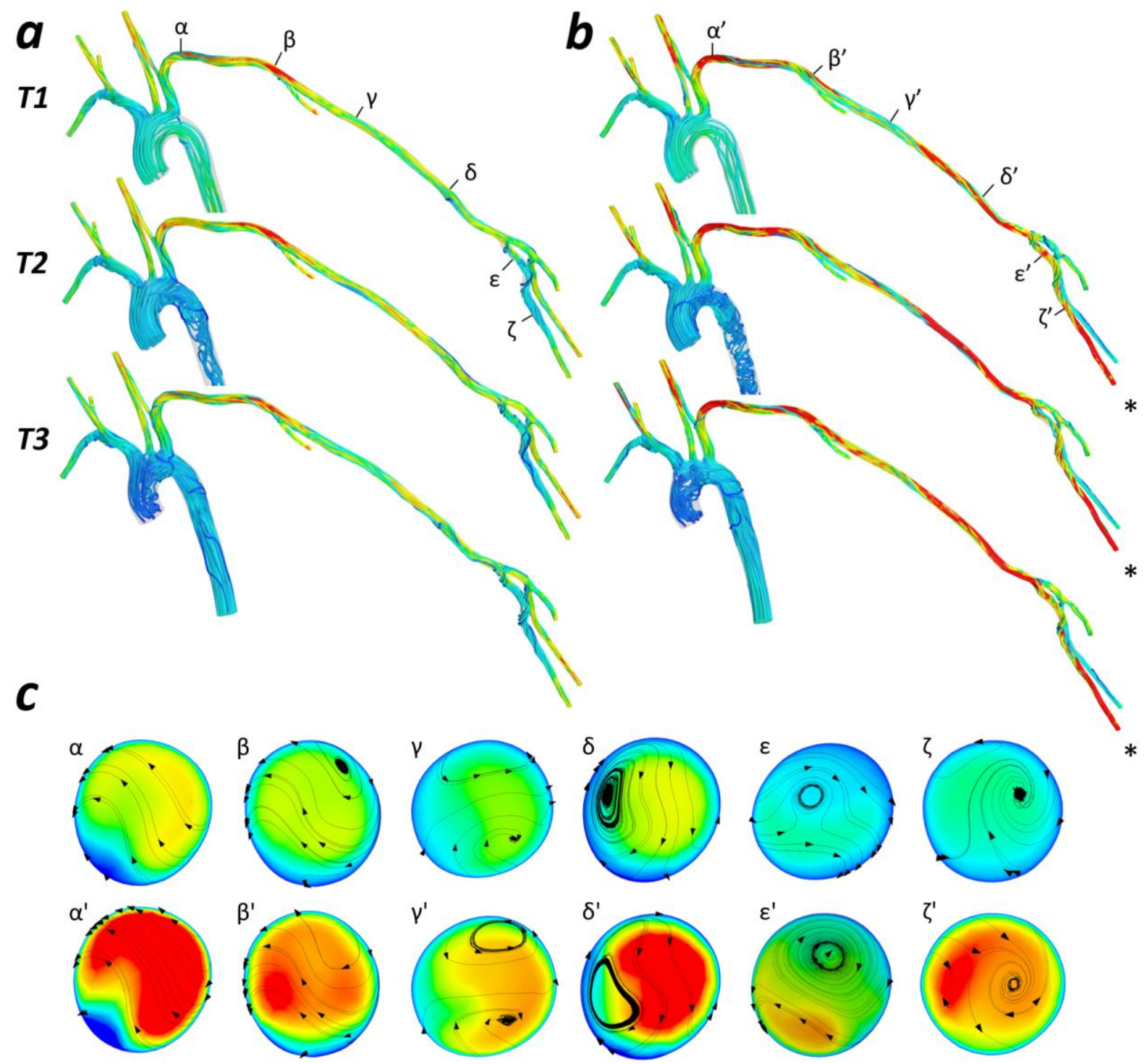

0.0 0.40

Velocity: Magnitude ( $\mathrm{m} / \mathrm{s})$

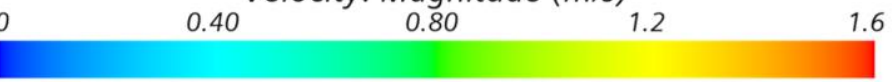

Figure 4: Velocity streamlines during the cardiac cycle (a) pre-AVF and (b) post-AVF at T1 (peak systole), T2 (mid-deceleration), and T3 (peak diastole). (c) Through-plane velocity contours overlaid by in-plane velocity vectors at six cross-sections along the left arm arteries, as shown in (a) and (b), at peak systole for pre- and post-AVF (top and bottom rows, respectively). Cross-sections are oriented such that top of each slice corresponds to the cranial edge of the lumen, looking upstream. The slices were positioned at 5D, 24D, 410D, 643D, 805D, and 900D downstream of the aortosubclavian junction, where $D$ is the left subclavian arterial diameter immediately following the aorto-subclavian junction. ${ }^{*}$ represents anastomosis location post-AVF 


\section{Haemodynamics at the AVF anastomosis}

The key metrics of interest related to AVF success are the venous diameter, venous flow rate, fistula depth, and fistula length (National Kidney Foundation's Kidney Disease Outcomes Quality Initiative (KDOQI) vascular access guidelines ${ }^{24}$ ). The depth of the fistula from the skin surface (using FeMRA) was found to be within the KDOQl's acceptable limits through analysis of the lower arm DICOM stack $(\leq 0.6 \mathrm{~cm})$. The fistula length (venous cannulation region) exceeded $6 \mathrm{~cm}$ and the venous diameter of the AVF was greater than $6 \mathrm{~mm}$ (Fig. 7). The non-physiological flow induced by the anastomosis exceeded $5 \mathrm{~m} / \mathrm{s}$ in the radial artery throughout the cardiac cycle and at the presented time intervals (Fig. 8a-c). In the cephalic vein, the velocity streamlines exhibited values below $2 \mathrm{~m} / \mathrm{s}$, however this equates to a flow rate in excess of $1000 \mathrm{ml} / \mathrm{min}$ (local Remax=3386). Downstream of the fistula, helical flow patterns were characteristically evident throughout the cardiac cycle, which persisted along the entire length of the cephalic vein. Fig. 8d shows the topology of instantaneous vortical structures at $\mathrm{T} 1$ as iso-contours of the Q-criterion (Supplementary Material), demonstrating the complexity of the flow field around the fistula. The percentage flow split between the distal arterial outlet (DAO) and the distal venous outlet (DVO) was 27-73\%. Fig. 8e-f presents the TAWSS and OSI distributions for the localised AVF geometry. Significantly high TAWSS values $(>120 \mathrm{~Pa})$ were observed in the feeding (radial) artery of the AVF, at the apex of the anastomosis, and on the venous side of the anastomosis. TAWSS progressively decreases along the venous and arterial outlet branches to much lower values at short distances from the fistula. Notably high OSI magnitudes (0.35-0.45) were observed in the distal artery branch of the AVF, in contrast to the negligible OSI values found upstream of the anastomosis. Moderately high OSI magnitudes were observed in the venous segment, however, these magnitudes occurred following the expansion of the venous segment to a crosssectional diameter of $6 \mathrm{~mm}$. 


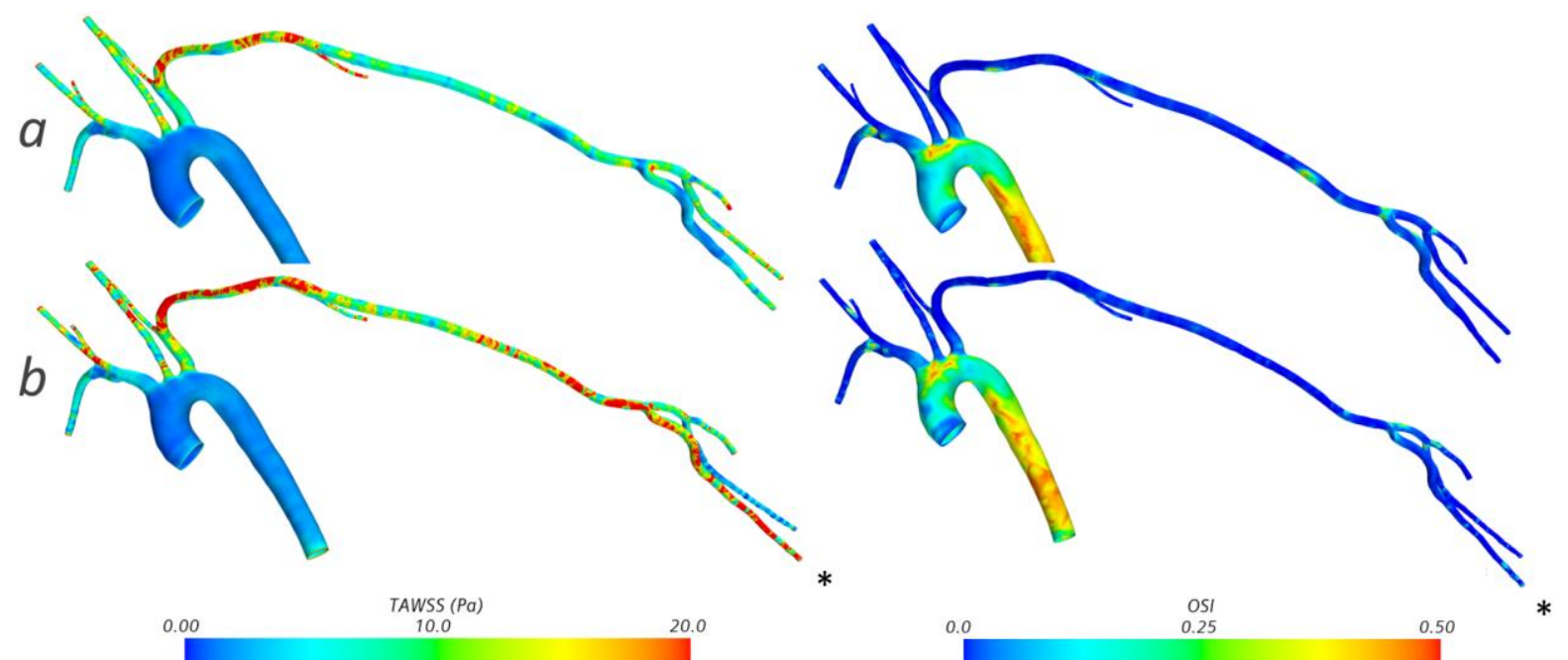

Figure 5: TAWSS (left) and OSI (right) contour plots prior-to (a) and after fistula creation (b). * represents anastomosis location

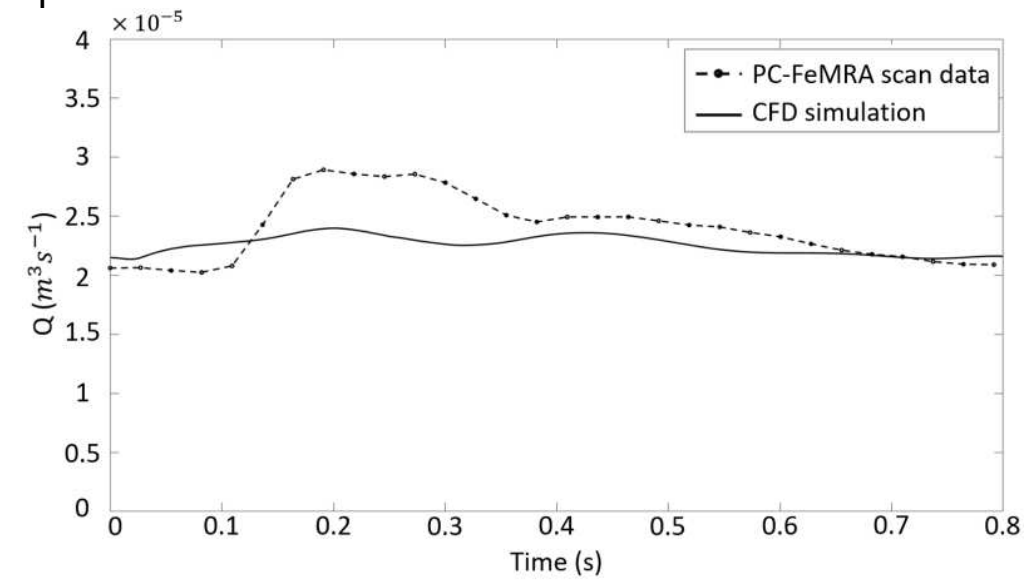

Figure 6: Comparison between the patient-specific phase-contrast FeMRA-obtained volumetric flow rate at the radial artery (slice $\varepsilon^{\prime}$ in Fig. 4c) and the waveform calculated using the CFD methods.

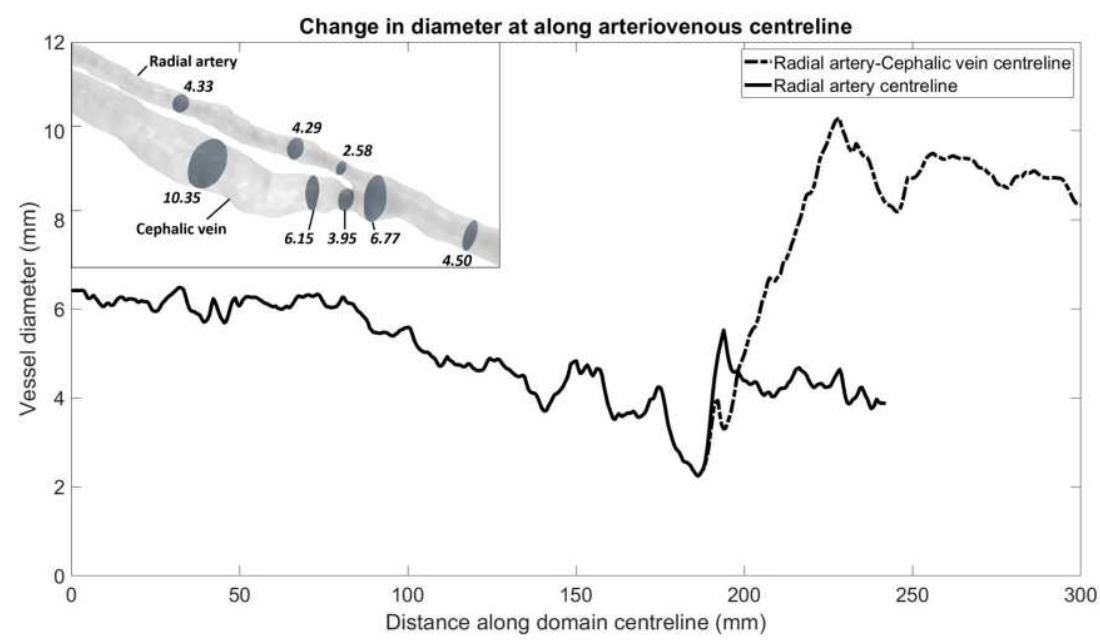

Figure 7: Geometry characterisation of the AVF juxta-anastomosis. 


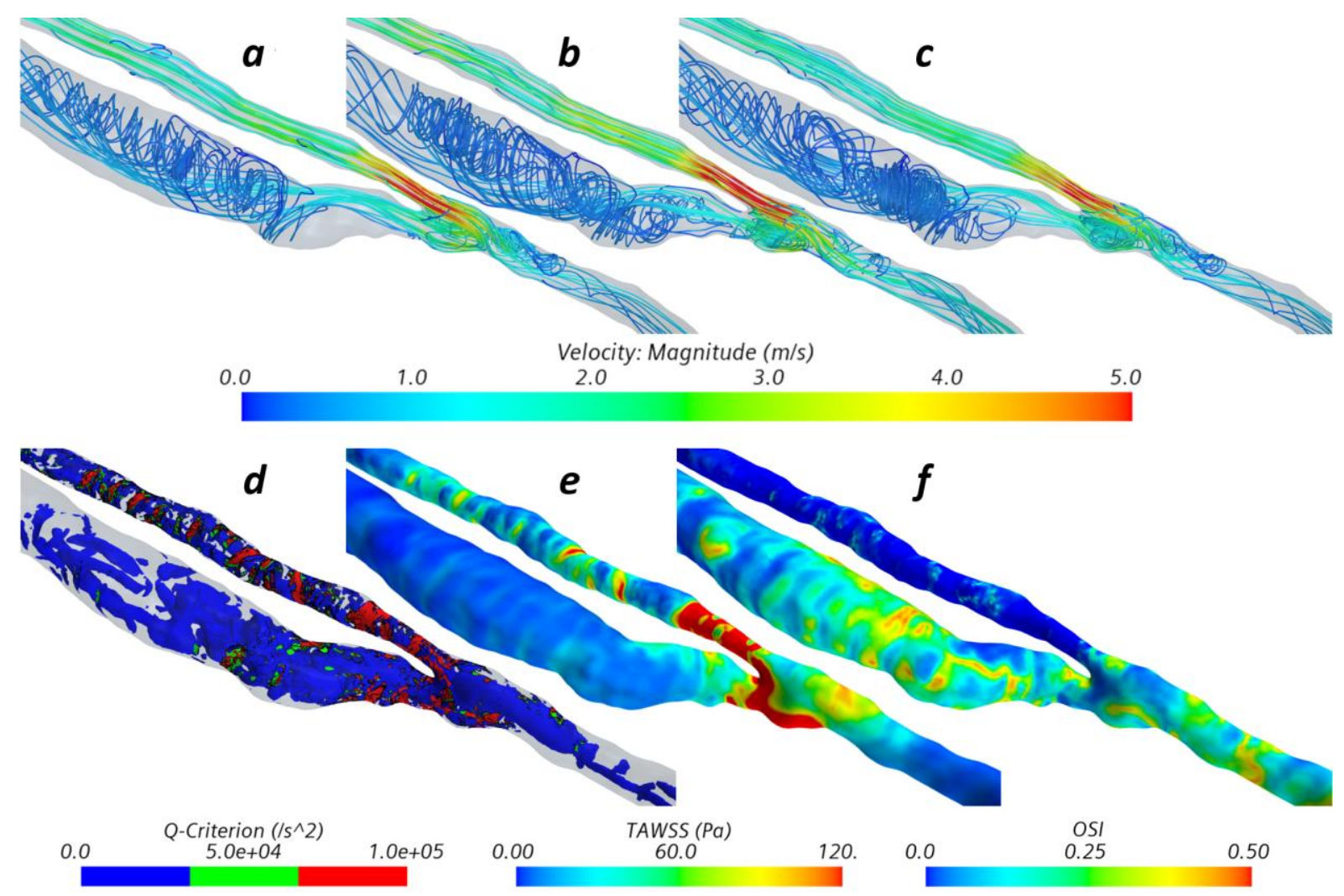

Figure 8: (a-c) Velocity streamlines in the radiocephalic fistula anastomosis at (a) T1 (peak systole), (b) T2 (mid-deceleration), and (c) T3 (peak diastole). (d) Instantaneous iso-contours of the Q-criterion at peak systole (T1), (e) TAWSS, (f) OSI.

\section{Discussion}

A framework for generating a patient-specific geometry from three high-resolution FeMRA scans of a young patient with CKD was implemented in this study for a CFD investigation with 3-element Windkessel boundary conditions. The dimensions of the generated domain were validated with respect to the original DICOM stacks. An investigation into the patient-specific geometry characteristics and the haemodynamics proximal to the AVF anastomosis, prior-to and after AVF creation was completed. This involved changing the specification of the radial artery pressure outlet condition between the physiological and non-physiological (AVF) cases to reflect the change in peripheral resistance induced from the creation of the AVF. The key finding was the marked increase in blood flow velocity and TAWSS demonstrated throughout the subclavian, brachial, and radial arteries in the arm of the radiocephalic AVF. This is a marker of adaptive arterial remodelling to accommodate the increased flow rates resulting from the surgical anastomosis. In addition to this, the geometry of the reconstructed AVF was examined, demonstrating a successful fistula maturation with respect to KDOQI guidelines. ${ }^{24}$ Moreover, the localised haemodynamic-based factors 
relating to $\mathrm{NH}$ and outward remodelling were analysed via CFD, confirming sufficient AVF flow rates $(>600 \mathrm{~mL} / \mathrm{min})$ and the generation of substantial helical flow patterns in the cephalic vein distal to the anastomosis throughout the cardiac cycle. This arises due to the significant change of direction of the flow entering the vein, which generates complex vortices and secondary flows when flow is exerted on the opposite vessel wall (Fig. 8). Our simulation indicated cyclic disturbed flow (OSI 0.42) in the radial artery distal to the anastomosis, and in the vein immediately following the venous swing segment of the AVF. This region of the vein, which experiences low and oscillatory shear stress, corresponds to where luminal reduction occurs due to $\mathrm{NH} .{ }^{12}$

\section{Limitations}

A limitation in this work was the assumption of rigid walls. However, this is a standard approach in the proximal-to-fistula case, since the influence of arterial wall deformation on the haemodynamic environment is considered relatively small in these vessels. For the juxta-anastomosis simulation, wall motion effects may be more pronounced. Fluidstructure interaction (FSI) methodologies can account for this behaviour, however, FSI modelling is more challenging and requires considerable computational expense. The assumption of Newtonian rheology in the proximal-to-fistula case is also a limitation, which is accepted in a large percentage of similar computational haemodynamic investigations. ${ }^{18,26}$ Furthermore, the utilisation of a polynomial triphasic aortic inlet waveform calculated from prior literature ${ }^{1}$ (due to unavailable patient-specific flow data at the ascending aorta) is a key assumption because of the influence of the boundary conditions on LES results. Also, a constant cardiac output was assumed between the pre- and post-AVF simulations. This assumption was eliminated in the juxtaanastomosis simulation with patient-specific phase-contrast data in the radial artery being used as the inlet waveform. Finally, the selection of Windkessel parameter values can also be considered a limitation due to the resistances and capacitances being estimated as per the 'Boundary conditions' subsection.

\section{Comparisons to prior work}

Prior work has been completed on coupling MRI and CFD ${ }^{17}$, and Bozzetto et al. ${ }^{5}$ demonstrated good quality images in a short scan duration when coupling contrastfree MRI and CFD. Similarly, good quality images were ascertained using ferumoxytol contrast in our presented framework. This study represents the first time FeMRA has 
been coupled with CFD, particularly regarding AVFs. Additionally, the majority of CFD studies on AVF haemodynamics focus on the local environment at the position of the anastomosis. ${ }^{15,37}$ For example, Cunnane et al. ${ }^{11}$ studied varying anastomosis angles and their influence on the WSS distribution, while Niemann et al ${ }^{26}$ examined WSS in six different fistula morphologies. In addition to analysing the local haemodynamics at the anastomosis, this study investigates, for the first time according to our knowledge, the blood flow dynamics proximal to the fistula. Caroli et al. ${ }^{8}$ reviewed the potential use of a patient-specific vascular low-order network model for predicting haemodynamic changes in the network six weeks after AVF creation. However, 3D numerical investigations, such as those presented in this paper, permits a more comprehensive analysis of the haemodynamics than low-order models, despite the inevitably increased computational expense, which is nonetheless lower than FSI simulations.

\section{Clinical relevance}

The benefit of using CFD in addition to FeMRA is the profiling of haemodynamic metrics that are difficult to measure in-vivo. This is highly relevant for the identification of probable sites of atherosclerotic lesion development proximal to the AVF, and the factors relating to peri-anastomotic intimal hyperplasia and inadequate outward remodelling at the outflow vein. The current consensus, particularly for adults and older ages, is that atherosclerosis usually occurs in arterial regions of low and/or oscillatory WSS. ${ }^{25}$ Furthermore, atherosclerotic development typically occurs at wall curvature locations and bifurcations, such as the brachiocephalic trunk, a known location for atherosclerotic lesion development that is also reflected in our results (Fig. 5). In addition to this, the increase in flow velocity and TAWSS at various locations post-AVF indicates likely arterial remodelling ${ }^{21}$ proximal to the location of the AVF. These markers were undermined in prior AVF studies, which focused predominately on localised AVF haemodynamics, or in some cases, the AVF's influence on cardiac load. The impact of arterial remodelling on the arm vessels proximal to the AVF may be detrimental to the patient through increased predisposition to other complications, such as cardiovascular disease and arterial steal syndrome. Further elucidation of important potential complications can be made using more thorough CFD with boundary conditions set by post-AVF patient flow data at several locations in the vasculature. The favourable hypothesis of high TAWSS causing outward remodelling 
for increased flow rates is also partially considered as a potential factor towards future endothelial damage. Despite the exact pathophysiology not being known, endothelial WSS triggers biomedical and biological events. Evidence suggests perturbed stress and strains disturb the biochemical homeostasis ${ }^{14}$ and cause adverse vascular remodelling and potential dysfunction. In addition to endothelial dysfunction, a harbinger of atherosclerosis, low WSS levels have been associated with an increase in neointima formation, and a proclivity toward vein vasoconstriction. ${ }^{23}$ However, $\mathrm{NH}$ lesions associated with AVFs differ from atherosclerotic lesions. $\mathrm{NH}$ occurs due to the proliferation of vascular smooth muscle cells, in addition to neovessels and inflammatory cells. Furthermore, optimal venous endothelial function has been associated with moderate/high WSS levels. The WSS levels modelled by CFD (Fig. 8) indicate the successful maturation of the AVF.

\section{Conclusions and further work}

As far as the authors are aware, this is the first study demonstrating the detailed dynamics of pulsatile blood flow from the aortic root to the distal arm arteries. For that purpose, a new methodological framework was presented involving the geometry reconstruction from multiple stacks of FeMRA-obtained medical images. This is an important step towards understanding the blood flow development proximal to the arm region where an AVF is created in ESKD patients. The last part of the study involved a detailed haemodynamic analysis of the flow around a patient-specific AVF. This is advantageous in the pre-surgical planning and post-operative evaluation of AVFs, as the arterial blood flow and cross-sectional diameter before AVF formation is known to correlate with its maturation. ${ }^{28}$ Additionally, the post-AVF venous blood flow rates relate to AVF maturation, hence analysis of the juxta-anastomosis haemodynamics becomes crucial in relation to success and failure rates. WSS is of high importance in this case, as outward remodelling occurs to reduce the WSS in the AVF over time. ${ }^{14}$ The remodelling is associated with the increase in vessel diameter required for the increased flow rates. Further work will seek to investigate the differing haemodynamic environments in a cohort of patients with varying AVF anastomoses configurations and outcomes (success vs failure). This will include brachiocephalic fistulae in addition to radiocephalic fistulae. Additionally, the arterial wall motion will be accounted for in future numerical simulations of the local AVF haemodynamics by incorporating an FSI element into the investigation. Several models and parameter definitions will be 
explored for modelling the hyperelastic nature of the arterial walls and venous segments. A methodology for refining the estimation of the characteristic Windkessel values applied at the outlets will also be implemented. Lastly, non-invasive techniques of atherosclerotic development, such as flow mediated dilation (FMD), will be explored in conjunction with AVFs. This approach will evaluate the potential of using computational modelling for simultaneously analysing the impact of FMD and AVF remodelling.

\section{Acknowledgments}

The authors greatly acknowledge the support from the Queen Elizabeth University Hospital (Glasgow, UK) and the University of Strathclyde.

\section{Funding}

This work is supported in part from the University of Strathclyde International Strategic Partner Research Studentships, and the EU H2020 research and innovation programme under the Marie Sklodowska-Curie grant agreement No 749185.

\section{Conflicts}

The authors declare no conflict of interest regarding the publication of this article.

\section{Ethics}

Patient-specific data presented here are from a previous prospective comparative study. ${ }^{34}$ The study protocol (http://dx.doi.org/10.36399/gla.pubs.215112) was approved by the institutional review board (Research Ethics Committee Reference: 16/NS/0099) and registered with ClinicalTrials.gov (NCT02997046). Written informed consent was obtained from all participants.

\section{References}

${ }^{1}$ Open source medical software corporation cpm specifications document, marfan: Osmsc 01740000, 2013.

${ }^{2}$ Vascular Access 2006 Work Group, Clinical practice guidelines for vascular access. Am J Kidney Dis 48, S176-S247, 2006. 
3 Alastruey Arimon, J. Numerical modelling of pulse wave propagation in the cardiovascular system: development, validation and clinical applications. PhD thesis, Imperial College London, UK, 2006.

${ }^{4}$ Boumpouli, M., et al. Blood flow simulations in the pulmonary bifurcation in relation to adult patients with repaired tetralogy of fallot. Med Eng Phys. 85:123-138, 2020.

${ }^{5}$ Bozzetto, M., et al. Toward longitudinal studies of hemodynamically induced vessel wall remodeling. Int J Artif Organs. 41 (11):714-722, 2018.

${ }^{6}$ Brescia, M. J., et al. Chronic hemodialysis using venipuncture and a surgically created arteriovenous fistula. N Engl J Med. 275(20):1089-92, 1966.

${ }^{7}$ Broadhead, B. L., et al. Sensitivity and uncertainty-based criticality safety validation techniques. Nucl Sci Eng. 146:3, 340-366, 2004.

${ }^{8}$ Caroli, A., et al. Validation of a patient-specific hemodynamic computational model for surgical planning of vascular access in hemodialysis patients. Kidney Int. 84(6):1237-45, 2013.

${ }^{9}$ Chnafa, C., et al. Better than nothing: A rational approach for minimizing the impact of outflow strategy on cerebrovascular simulations. Am J Neuroradiol. 39(2):337-343, 2018.

${ }^{10}$ Cockwell, P., and Fisher, L. The global burden of chronic kidney disease. The Lancet. 395(10225):662-664, 2020.

${ }^{11}$ Cunnane, C. V., et al. The presence of helical flow can suppress areas of disturbed shear in parameterised models of an arteriovenous fistula. Int $\mathrm{J}$ Numer Method Biomed Eng. 35(12):e3259, 2019.

12 Enelordache, B., and Remuzzi, A. Disturbed flow in radialcephalic arteriovenous fistulae for haemodialysis: low and oscillating shear stress locates the sites of stenosis. Nephrol. Dial. Transplant. 27(1): 358-368, 2011. 
${ }^{13}$ Feldman, H. I., et al. Hemodialysis vascular access morbidity. Clin J Am Soc Nephrol. 7(4):523-35, 1996.

${ }^{14}$ Gameiro, J., and Ibeas, J. Factors affecting arteriovenous fistula dysfunction: A narrative review. J Vasc Access. 21(2):134-147, 2020.

${ }^{15}$ Grechy, L., et al. Suppressing unsteady flow in arteriovenous fistulae. Phys Fluids. 29, 101901, 2017.

${ }^{16}$ Guyton, A. C., and Sagawa, K. Compensations of cardiac output and other circulatory functions in areflex dogs with large av fistulas. Am. J. Physiol. Legacy Content 200:1157-63, 1961.

${ }^{17} \mathrm{Ha}, \mathrm{H}$., et al. Fluid-dynamic optimal design of helical vascular graft for stenotic disturbed flow. PLOS ONE 9(10):e111047, 2014.

${ }^{18}$ Johnston, L., et al. Hemodynamic abnormalities in the aorta of turner syndrome girls. Front. Cardiovasc. Med. 8:670841, 2021.

${ }^{19}$ Kazakidi, A., et al. Effect of reynolds number and flow division on patterns of haemodynamic wall shear stress near branch points in the descending thoracic aorta. J R Soc Interface. 6(35):539-48, 2008.

${ }^{20}$ Kazakidi, A., et al. Effect of reverse flow on the pattern of wall shear stress near arterial branches. J R Soc Interface. 8(64):1594-603, 2011.

${ }^{21}$ Krishnamoorthy, M., et al. Hemodynamic wall shear stress profiles influence the magnitude and pattern of stenosis in a pig av fistula. Kidney Int. 74(11):1410-9, 2008. ${ }^{22}$ Kumar, A., et al. Radiomedian cubital / radiocephalic arteriovenous fistula at elbow to prevent vascular steal syndrome associated with brachiocephalic fistula: Review of 320 cases. Indian J Urol. 23(3):261-4, 2007.

23 Lehoux, S., et al. Molecular mechanisms of the vascular responses to haemodynamic forces. J. Intern. Med. 259(4):381-92, 2006. 
${ }^{24}$ Lok, C. E., et al. Kdoqi clinical practice guideline for vascular access: 2019 update. Am J Kidney Dis. 75(4 S2):S1-S164, 2020.

25 Mitchell, J. R. A., and Schwartz, C. J. Arterial disease. Blackwell Scientific Publications, 17(2):69-71, 1965.

${ }^{26}$ Niemann, A., et al. Computational fluid dynamics simulation of av fistulas: From mri and ultrasound scans to numeric evaluation of hemodynamics. J Vasc Access. 1 3(1):36-44, 2011.

${ }^{27}$ Okada, S., and Shenoy, S. Arteriovenous access for hemodialysis: Preoperative assessment and planning. J Vasc Access. 15(S7):S1-5, 2014.

${ }^{28}$ Oprea, A., et al. Correlation between preoperative vein and artery diameters and arteriovenous fistula outcome in patients with endstage renal disease. Clujul Med. 91(4):399-407, 2018.

${ }^{29}$ Papageorgiou, G. L., and Jones, N. B. Wave reflection and hydraulic impedance in the healthy arterial system: a controversial subject. Med Biol Eng Comput. 26(3):23742, 1988.

${ }^{30}$ Polkinghorne, K. R., et al. Vascular access and all-cause mortality: A propensity score analysis. Clin J Am Soc Nephrol. 15(2):477-86, 2004.

${ }^{31}$ Robbin, M. L., et al. Arteriovenous fistula development in the first 6 weeks after creation. Radiology 279(2):620-9, 2016.

32 Roy-Chaudhury, P., et al. Predicting dialysis vascular access blood flow and diameter: too much, too little, or just right. Kidney Int. 84(6):1076-1078, 2013.

${ }^{33}$ Stoumpos, S., et al. Ferumoxytol-enhanced magnetic resonance angiography for the assessment of potential kidney transplant recipients. Eur Radiol. 28(1):115-123, 2018. 
${ }^{34}$ Stoumpos, S., et al. Ferumoxytol mr angiography versus duplex us for vascular mapping before arteriovenous fistula surgery for hemodialysis. Radiology 297(1):200069, 2020.

${ }^{35}$ Van Doormaal, M., et al. Haemodynamics in the mouse aortic arch computed from mri-derived velocities at the aortic root. J R Soc Interface 9(76):2834-44, 2012.

${ }^{36}$ Wong, C., et al. Vascular remodeling and intimal hyperplasia in a novel murine model of arteriovenous fistula failure. J Vasc Surg. 59(1):192-201, 2013.

37 Yang, C., et al. The anastomotic angle of hemodialysis arteriovenous fistula is associated with flow disturbance at the venous stenosis location on angiography. Front. Bioeng. Biotechnol. 8:846, 2020.

${ }^{38}$ Yushkevich, P., et al. Itk-snap: An interactive tool for semiautomatic segmentation of multimodality biomedical images. $38^{\text {th }}$ Ann. Int. Conf. IEEE Eng. Med. Biol. Soc. pp.3342-3345, 2016.

${ }^{39} \mathrm{Zhu}, \mathrm{Y}$., et al. Clinical validation and assessment of aortic hemodynamics using computational fluid dynamics simulations from computed tomography angiography. Biomed. Eng. Online 17:53, 2018. 


\section{Supplementary Files}

This is a list of supplementary files associated with this preprint. Click to download.

- SupplementaryMaterialFinal.pdf 